\title{
MAST CELLS DEPLETION IMPROVES BONE MARKERS EXPRESSION ON MANDIBLE OF SPONTANEOUSLY HYPERTENSIVE RATS WITH PERIODONTAL DISEASE
}

\author{
Sandra Oliveira ${ }^{1,2}$, Victor Brito ${ }^{1,2}$, Ayna Barreto ${ }^{1,2}$, Mariana Patrocinio ${ }^{1}$, Maria Carolina Sousa ${ }^{1}$, \\ Carluci Beltan ${ }^{1,2}$, Dayane Queiroz ${ }^{1,2}$, Leticia Vieira ${ }^{1,2}$, Vanessa Lara ${ }^{4}$, Carlos Santos ${ }^{3}$ \\ ${ }^{1}$ Basic Science, São Paulo State University (UNESP), School of Dentistry, Brazil, ${ }^{2}$ Multicenter Graduate Program in \\ Physiological Sciences, SBFis, Brazil, ${ }^{3}$ Department of Biological Sciences, Bauru School of Dentistry, University of São \\ Paulo, Brazil, ${ }^{4}$ Department of Oral Surgery, Stomatology, Pathology and Radiology, Bauru School of Dentistry, \\ University of São Paulo, Brazil
}

Background

Periodontal disease (PD) is an inherited or acquired disorder of tooth surrounding tissues and alveolar bone initiated by bacteria biofilm accumulation. Coexistence of systemic conditions, such as hypertension, can lead to exacerbated inflammatory response and enhanced bone resorption. Resident immune cells have a crucial role in PD progression however mast cells participation is not yet well comprehended.

Aims

Evaluate the role of mast cells on gene expression of bone metabolism on mandibles of normotensive and hypertensive rats with experimentally induced PD.

Methods

10 week old male Wistar and SHR were pre treated with 48/80 compound and subjected to 15 days of PD induced by bilateral silk ligature placed on the first inferior molars. Experimental groups were labeled as Control (C), PD and 48/80 + PD of Wistar (W) and SHR (S) animals. Hemimandibles were harvested for microCT analysis and real time RT PCR assays of bone formation markers: Runx2, Osterix, Beta catenin, osteoprotegerin, BMP 2, alkaline phosphatase, osteocalcin, osteopontin and bone sialoprotein and bone resorption remodeling markers: tartrate resistant acid phosphatase, RANK RANKL, cathepsin K, MMP 2 and 9 and OSCAR. The protocol was approved by Institutional Animal Care and Use Committees School of Dentistry of Aracatuba (Process 00686/2016).

Results

MicroCT showed increased alveolar bone loss on SPD which was reduced by mast cell depletion. Gene expression of bone formation markers did not reveal major alterations except for Opn which was significantly increased in PD groups. However, bone resorption markers Trap, Mmp9, Ctsk and Oscar showed higher expression on SC compared with WC. PD groups showed higher expression of those markers compared with C group especially on SPD which had further increased resorption markers expression. Mast cells depletion previously to PD induction was able to reduce those resorption markers and interestingly it was more significant on hypertensive animals S+48 80+PD.

Conclusion

Mast cells may have an important role in bone resorption markers expression mainly in hypertensive animals with PD with possible participation in alveolar destruction. These are preliminary results and other experimental analyses are been executed to find further evidence for our better understanding of this effects. 